\title{
Determination of radius of the black hole
}

\author{
Sergey Orlov \\ Petrozavodsk State University, c. Petrozavodsk, Russia
}

Email address:

ion@sampo.ru

\section{To cite this article:}

Sergey Orlov. Determination of Radius of the Black Hole. American Journal of Astronomy and Astrophysics. Vol. 2, No. 2, 2014, pp. 18-21. doi: 10.11648/j.ajaa.20140202.11

\begin{abstract}
Offered a theoretical calculation of the radius of the Black Hole, based on vortex theory of gravity. Proposed substantiation that the volume of the Black Hole independent of the mass or density of its material. Shown conflicting decisions on the definition of the radius of the Schwarzschild Black Hole, which prove that this decision can not be taken for scientific research.
\end{abstract}

Keywords: Theory of Vortex Gravitation, Cosmology, Cosmogony

\section{Schwarzschild 'S Decision}

Black hole' - area in a space which gravitational attraction is so great that the objects moving with velocity of light including quanta of light can't leave it even. The border of this area is called as gravitational radius. Spherically a symmetric black hole it is equal in the elementary case to Shvwarzshild's radius.

Theoretically possibility of existence of such areas of space-time follows from some exact solutions of the equations of Einstein, first of which was received by Charles Shvwarzshild in 1915 y. [1].

$$
r_{s}=\frac{2 G M}{c^{2}}
$$

Where $r_{s}$ - the radius of the Black Hole, $M-$ the mass of a black hole, $c$ - the velocity of light, $G=6.672 \cdot 10^{-11}$ $\mathrm{N} \cdot \mathrm{m} 2 / \mathrm{kg} 2$ - a gravitational constant.

Shvwarzshild 's decision is based on a well-known classical hypothesis that all bodies create attraction forces. The hypothesis of the world attraction never had sufficient proofs for the existence. Therefore, throughout centuries, the concept about gravitational properties of physical bodies is accompanied by a set of unsoluble paradoxes. Shvwarzshild 's decision, based on this inconsistent theory hypothesis, generates the paradoxes. In particular, in a formula (1) physical discrepancy is put - the radius of the Black Hole (sphere) is directly proportional to the mass of this object. It is well-known that the mass of any body is directly proportional to the volume of this body. In turn, the volume of a body (sphere) is proportional to a cube of radius of this body. Therefore, the mass of a body can be in directly proportional dependence on a cube of radius of this body, instead of on radius, as in a formula (1).

This discrepancy generates absurd results at settlement use of the equation (1). In particular, at theoretical determination of radius of the Black Hole with a weight to the equal mass of our mother Earth, density of substance of this Black Hole has to be about $10^{30} \mathrm{~kg} / \mathrm{m}^{3}$ that considerably exceeds density of an atomic nucleus!? As it is known in the center of our galaxy there is a Black Hole (BH). In article [2] the radius of this $\mathrm{BH}-\mathrm{R}_{\mathrm{BH}}=6,75 \mathrm{x}$ $10^{12} \mathrm{~m}$ is determined. But at such radius and, the corresponding volume, the mass of this $\mathrm{BH}$ has to be on the basis of a formula (1) order $10^{39}$ кг. Then density of the Black Hole in the center of our galaxy has to be as only 5 $\mathrm{kg} / \mathrm{m}^{3} ! ?$

Assumption No 1. The hypothetical Black Hole $\mathrm{R}_{\mathrm{bh}}=10^{20}$ $\mathrm{M}$ radius, has to have density of the substance, on the basis of a formula (1), as $10^{-14} \mathrm{~kg} / \mathrm{m} 3$ ?! But such density is less than density of ether. Therefore, Shvwarzshild 's equation (1) is absurd.

Below determination of radius of $\mathrm{BH}$ on the basis of the theory of vortex gravitation which doesn't contain any contradictions is offered.

\section{On the Theory of Vortex Gravitation}

The theory of vortex gravitation [3] is based on the wellknown astronomical fact - all heavenly objects rotate. The most logical explanations of the reason of this movement can be only one - rotation of heavenly objects generated vortex rotation of space substance - ether. Ether forms 
system of interconnected whirlwinds in world space. Orbital speeds of ether in each tuft (torsion) decrease in the direction from the center to the periphery under the law of the return square of this removal. If orbital speeds of a stream of ether decrease then, under aerodynamics laws, pressure in this stream increases. The gradient of pressure generates pushing out force in the direction to zones with the smallest pressure, that is to the center of this torsion. Thereby in the center of the torsion the space substance collects and the heavenly body is created. Bodies or the substances moving on orbits of the torsion with the $1 \mathrm{st}$ space speed, become constant satellites of this space system (torsion).

Let's consider the equation of vortex gravitation received in the theory [3].

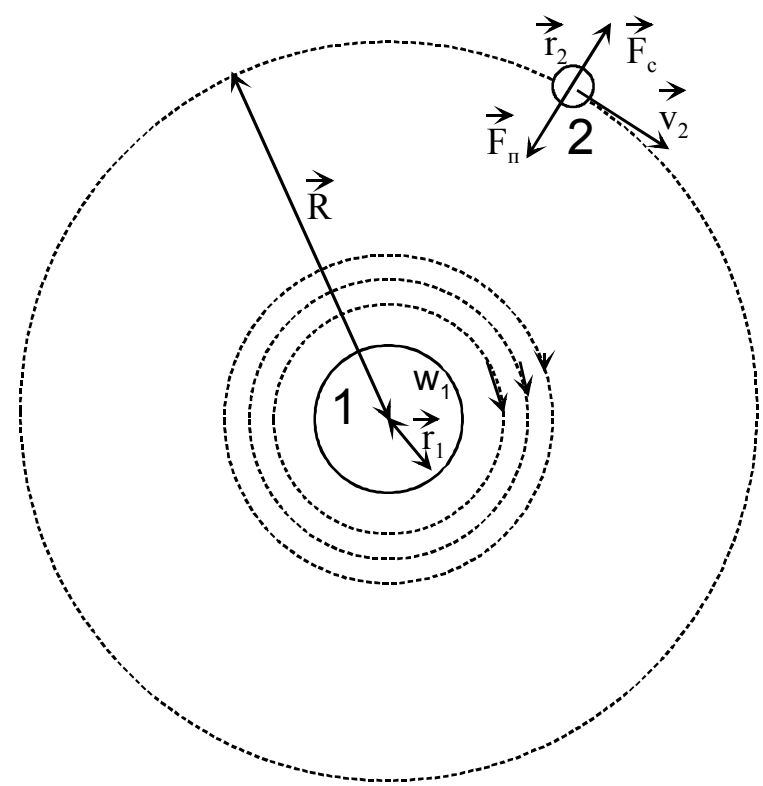

Fig 1. Two-dimensional model of gravitational interaction of two bodies. The forces are shown acting on body $2: F_{c}$-the centrifugal force, $F_{n}-$ the force of attraction of body 2 from body $1 ; v_{2}$-linear velocity of body 2 at the orbit, $r$-the radius of the orbit, $r_{1}$ - the radius of body $1, r_{2}$-the radius of body 2, $w_{1}$-angular velocity of ether rotation at the surface of body 1 , and $m_{2}$ are the mass of body 2 .

Next we consider the appearance of the attraction force in more detail and derive a formula describing it. As was said above, a pressure gradient arises as the result of the vortex motion. Let's find the radial distribution of the pressure and the ether velocity. For this purpose, we write the Navier-Stokes equation for the motion of a viscous liquid (gas).

$$
\rho\left[\frac{\partial}{\partial t}+\vec{v} \cdot \operatorname{grad}\right] \vec{v}=\vec{F}-\operatorname{grad} P+\eta \Delta \vec{v}
$$

where $\rho$ is the ether density, $\vec{V}$ and $P$ are, respectively, its velocity and pressure, and $\eta$ - the ether viscosity. In cylindrical coordinates, taking into account the radial symmetry $\mathrm{v}_{\mathrm{r}}=\mathrm{v}_{\mathrm{z}}=0, \mathrm{v}_{\varphi}=\mathrm{v}(\mathrm{r}), \mathrm{P}=\mathrm{P}(\mathrm{r})$, the equation can be written as the system:

$$
\left\{\begin{array}{l}
-\frac{\mathrm{v}(\mathrm{r})^{2}}{\mathrm{r}}=-\frac{1}{\rho} \frac{d \mathrm{P}}{d \mathrm{r}} \\
\eta \cdot\left(\frac{\partial^{2} \mathrm{v}(\mathrm{r})}{\partial \mathrm{r}^{2}}+\frac{\partial \mathrm{v}(\mathrm{r})}{\mathrm{r} \partial \mathrm{r}}-\frac{\mathrm{v}(\mathrm{r})}{\mathrm{r}^{2}}\right)=0
\end{array}\right.
$$

In case of a compressible substance (ether), there will be a function $\rho=f(P)$ (instead of $\rho$ ).

From the first equation of system (3), one can find $\mathrm{P}(\mathrm{r})$ provided that the dependence $\mathrm{v}(\mathrm{r})$ is known. The latter, in turn, should be found from the second equation of that same system (one of the solution of which is the function $\mathrm{v}(\mathrm{r}) \sim 1 / \mathrm{r})$. At zero viscosity, the system permits any dependence v(r) [5].

The force affecting the body can be estimated from the formula

$$
\overrightarrow{\mathrm{F}}_{\text {п }}=-\mathrm{V} \cdot \operatorname{grad} \mathrm{P}(\mathrm{r})
$$

where $\mathrm{V}$ is the volume of body 2 .

In cylindrical coordinates the modulus of $\overrightarrow{\mathrm{F}}_{\Pi}$ is

$$
\mathrm{F}_{\text {п }}=\mathrm{V} \cdot \frac{\partial \mathrm{P}}{\partial \mathrm{r}}
$$

Then, comparing equations (3) and (5), for the incompressible ether $(\rho=$ const $)$ we find that

$$
\mathrm{F}_{\Pi}=\mathrm{V} \cdot \rho \cdot \frac{\mathrm{V}(\mathrm{r})^{2}}{\mathrm{r}}
$$

For the correspondence of the ether rotation to the planet motion law (according to Kepler 3-rd law) in one cosmic (e.g., Solar) system, v(r) must obey the dependence $\mathrm{v}(\mathrm{r}) \sim \frac{1}{\sqrt{\mathrm{r}}}$, and not the $\mathrm{v}(\mathrm{r}) \sim \frac{1}{\mathrm{r}}$.

Taking into account the edge condition $\mathrm{v}\left(\mathrm{r}_{1}\right)=\mathrm{w}_{1} \cdot \mathrm{r}_{1}$,

$$
\mathrm{v}(\mathrm{r})=\frac{\mathrm{w}_{1} \cdot r_{1}^{\frac{3}{2}}}{\sqrt{\mathrm{r}}}
$$

Thus

$$
\mathrm{F}_{\mathrm{\Pi}}=\mathrm{V} \cdot \rho \cdot \frac{w_{1}^{2} \cdot r_{1}^{3}}{\mathrm{r}^{2}}
$$

Here we make one more supposition (№ 6) - Ether penetrates through all the space, including the physical bodies. The volume V in formula (8) is an effective volume, i.e. the volume of elementary particles, which the body is composed of. All the bodies are composed of electrons, protons, and neutrons. The radius of an electron is much smaller than that of a proton and neutron. The radii of the latter are approximately equal to each other, $\mathrm{r}_{\mathrm{n}} \sim 1.2 \cdot 10^{-15} \mathrm{~m}$. 
The same is true as to the masses: $\mathrm{m}_{\mathrm{n}} \sim 1.67 \cdot 10^{-27} \mathrm{~kg}\left(\mathrm{r}_{\mathrm{n}}\right.$ and $\mathrm{m}_{\mathrm{n}}$ are the radius and the mass of a nucleon). Therefore, the volume in formula (8) is:

$$
\mathrm{V}=\frac{\mathrm{m}_{2}}{\mathrm{~m}_{\mathrm{n}}} \cdot \frac{4 \pi}{3} \cdot \mathrm{r}_{\mathrm{n}}{ }^{3}
$$

Taking into account the formula (9), Eq. (8) can be rewritten as

$$
\mathrm{F}_{\Pi}=\frac{4 \cdot \pi \cdot \mathrm{r}_{\mathrm{n}}^{3} \cdot \rho}{3 \cdot \mathrm{m}_{\mathrm{n}}} \cdot \frac{w_{1}^{2} \cdot r_{1}^{3} \cdot \mathrm{m}_{2}}{\mathrm{r}^{2}}
$$

where

$$
p \sim 8.85 \times 10^{-12} \mathrm{~kg} \backslash M^{3} \text { - ether density [4], }
$$

Let's transform a formula (10). Let's equate $r_{1}=r$. Let's substitute $\mathrm{w}_{1} \mathrm{X}_{1}=\mathrm{v}_{1}$ and numerical values $\mathrm{r}_{\mathrm{n}}, \mathrm{m}_{\mathrm{n}}, \rho$ we receive:

$$
\mathrm{F}_{\text {п }}=3.83 \times 10^{-29} \times V_{1}^{2} \times m / r
$$

For the circulation of the inertial body around the torsion center with the first space, a constant in absolute speed, you have to satisfy the conditions equal force of gravity $\mathrm{F} n$ and centrifugal force Fc, acting on the body, that is,

$$
\mathrm{F}_{\text {п }}=\mathrm{F}_{\text {п }}
$$

or

$$
3.83 \times 10^{-29} \times V_{1}^{2} \times m / r=V^{2} \times m / r
$$

whence

$$
3.83 \times 10^{-29} \times V_{1}^{2} \times m / r=V^{2}
$$

or

$$
V_{1}=5.11 \times 10^{14} \times V
$$

where

$\mathrm{V}$ - speed of the studied body moving on an orbit of the torsion at which air addresses with a speed $-\mathrm{v}_{1}$.

The equation (12) shows that for achievement of the first space speed the body has to move round the torsion center with a speed of $5,11 \times 10^{14}$ times less than ether speed in this orbit.

Note. According to the theory of vortex gravitation force of gravity produce essential torsion bars, and not the body (matter). Each torsion bar at its center collects cosmic substance. Therefore it is necessary to recognize that the amount of matter (mass or volume) of a space object has a dependency on the strength of the vortex gravitation. To determine the mass or density of a celestial object requires different information about this celestial object. In particular, the amount of a substance that absorbs a cosmic torsion depends on the period of existence of this ether torsion. The longer the torsion bar, the more he accumulated cosmic matter in its gravitational field, and the denser the material will be. Therefore, to determine the mass or density of celestial objects requires further investigation. The density of the Black Hole can not be determined, as due to the effect of confinement of the light flux is not available to us no information about the internal structure of the Black Hole.

On the basis of the theory of a vortex cosmogony [3], Black holes it is an initial stage of existence of the space torsion. After accumulation in the torsion of space substance in volume bigger, than the volume of the Black Hole, this Black Hole turns into a star.

\section{Vortex Black Hole}

According to the theory of vortex gravitation, the force of gravity creates a vortex rotation of the ether, and not the mass of the body, which is located in the center of torsion. Consequently, low-orbiting speed ether (V1c) reaches a value when no body or substance (including light) can not come off the orbit. This speed is determined by the equation (12):

$$
\begin{aligned}
& V_{1 c}=5.11 \times 10^{14} \times c \\
& =5.11 \times 10^{14} \times 3.0 \times 10^{8} \\
& =1.53 \times 10^{23}
\end{aligned}
$$

where

$$
C=3 \times 10^{8} m \backslash c-\text { speed of light. }
$$

Using the inverse square of the orbital velocity of the ether on the diameter of the orbit at which the ether is drawn - v(r) $\frac{1}{\sqrt{r}}$ obtain an equation for determining the radius of the black hole $-\mathrm{RBH}$.

$$
R_{b h}=r /\left(V_{1 c} / V_{1}\right)^{2}
$$

where

$r$ - radius of the orbit at which the appeals body (satellite),

$\mathrm{V}_{1}$ - velocity of the ether in an orbit with a radius $\mathrm{r}$.

$\mathrm{V}_{1 \mathrm{c}}=1,53 \times 10^{23}$ - ether velocity in orbit, on which the speed of light corresponds to the first cosmic velocity, according to (13).

We define the radius of the black hole sun torsion, using the known values:

The radius of the Earth's orbit $\mathrm{r}=1,5 \times 10^{11}$, orbital speed of the Earth $\mathrm{V}=30000 \mathrm{~m} / \mathrm{s}$, ether velocity in the orbit of the Earth around the Sun, according to the formula 12 ,

$$
V_{1}=3 \times 10^{4} \times 5.11 \times 10^{14}=1.53 \times 10^{19}
$$

ether velocity in orbit treatment of light (Eq. 13) 
$V_{1 c}=1.53 \times 10^{23}$,

Substituting into equation $r, V_{1}$ and $V_{1 c}$ in equation (14)

$$
R_{b h s}=1.5 \times 10^{11} \times\left(1.53 \times 10^{23} / 1.53 \times 10^{19}\right)=1500 M
$$

We define the radius of the black hole in our galaxy on the following grounds:

The orbital speed of the solar system $V=12.3 \times 10^{5}$ [6], ether velocity in solar orbit, based on equation (12) is

$$
V_{1}=2.3 \times 10^{5} \times 5.11 \times 10^{14}=1.18 \times 10^{20}
$$

the radius of the orbit of the solar system $r=2.46 \times 10^{20}$ [5].

The radius of the orbit of the black hole in our galaxy (Equation 14):

$$
\begin{aligned}
R_{b h s} & =2.46 \times 10^{20} /\left(1.53 \times 10^{23} / 1.18 \times 10^{20}\right)^{2} \\
& =1.45 \times 10^{14} M
\end{aligned}
$$

On the basis of the equation Schwarzschild radii identified other black holes:

- For the sun torsion $R_{B H}=3000 m$

- For galactic $R_{B H}=6.75 \times 10^{12} \mathrm{~m}$

\section{Conclusion}

In article it is shown that determination of physical parameters of Black Holes by means of the equation received from the classical theory of gravitation, leads to absurd results. It is explained only by that widely known theories of gravitation are based on a wrong hypothesis in which it is unsubstantially claimed that all bodies have ability to attract each other.
The theory of vortex gravitation is based on other law of a space substance - gravitation is created by the space environment (ether). Mass or density of the Black Hole can not be determined, since its mass is determined not only by the power of its own gravity, but also a lifetime of this celestial object. The midget density of space ether complicates comprehensive studying of its properties and laws. But results which are received by means of the equations of vortex gravitation, are relieved of any contradictions and therefore deserve bigger attention in scientific researches.

\section{References}

[1] K. Schwarzschild Über das Gravitationsfeld eines Massenpunktes nach der Einsteinschen Theorie // Sitzungsberichte der Königlich Preussischen Akademie der Wissenschaften 1. - 1916. - 189-196.

[2] Downes, D.; Martin, A. H. M. Compact Radio Sources in the Galactic Nucleus // Nature. - 1971. - Vol. 233. C. $112-114$.

[3] S. Orlov. Foundation of vortex gravitation, cosmology and cosmogony. Global journal of science Frontier research. Physic and Space Science

[4] V. A. Atsurovskiy. General ether-dynamics. Energoatomizdat. Moscow, Russia. 1990. Page 278.

[5] L. V. Kiknadze, Yu G Mamaladze. Classical hydrodynamics for physicists-experimentalists. Tbilisi University Press. Tbilisi, Georgia. 1979. Page 136.

[6] Balick, Bruce; Sanders, Robert H. Radio Fine Structure in the Galactic Center // Astrophysical Journal. — 1974. Vol. 192 